\title{
Nanometer-scale Tomographic Reconstruction of 3D Electrostatic Potentials in GaAs/AlGaAs Core-Shell Nanowires
}

\author{
A. Lubk, D. Wolf, S. Sturm, and H. Lichte \\ Triebenberg Laboratory, Institute of Structure Physics, \\ Technische Universität Dresden, 01062 Dresden, Germany \\ P. Prete \\ IMM-CNR, Lecce Research Unit, S.P. 6 Lecce-Monteroni, I-73100, Lecce, Italy \\ N. Lovergine \\ Dipartimento di Ingegneria dell'Innovazione, Università del Salento, \\ S.P. 6 Lecce-Monteroni, I-73100 Lecce, Italy \\ T. Niermann \\ Institut für Optik und Atomare Physik, Technische Universität Berlin, \\ Straße des 17. Juni 135, 10623 Berlin, Germany
}

We report on the development of Electron Holographic Tomography towards a versatile potential measurement technique, overcoming several limitations, such as a limited tilt range, previously hampering a reproducible and accurate electrostatic potential reconstruction in three dimensions. Most notably, tomographic reconstruction is performed on optimally sampled polar grids taking into account symmetry and other spatial constraints of the nanostructure. Furthermore, holographic tilt series acquisition and alignment have been automated and adapted to three dimensions. We demonstrate $6 \mathrm{~nm}$ spatial and $0.2 \mathrm{~V}$ signal resolution by reconstructing various, previously hidden, potential details of a GaAs/AlGaAs core-shell nanowire. The improved tomographic reconstruction opens pathways towards the detection of minute potentials in nanostructures and an increase in speed and accuracy in related techniques such as X-ray tomography.

Tomographic techniques use lower dimen- matician J. Radon [1] and to date applications sional projections of $n$-dimensional data to re- in fields as diverse as medicine, geophysics, maconstruct the original quantity. This principle terial science and quantum information are redates back to the work of the Austrian mathe- ported. Our main goal is the tomographic re- 
construction of $3 \mathrm{D}$ electrostatic potentials in nanostructures. They are tightly connected to the chemical composition and electronic structure and therefore mirror the corresponding functionality and possible failures. Off-axis electron holography (EH) provides unique access to these fields [2-4] because the reconstructed phase is in the phase grating approximation proportional to the potential projected along lines $l$

$$
\varphi(x, p)=\left(C_{E} \int_{l(x, p, \theta)} V(\mathbf{r}) \mathrm{d} s\right) \bmod (2 \pi)
$$

which holds for a wide range of imaging conditions [5]. Here, $C_{E}$ denotes the electronmatter interaction constant depending only on the acceleration voltage of the electrons $\left(C_{E}=\right.$ $6.5 \mathrm{mrad} /(\mathrm{Vnm}) @ 300 \mathrm{kV}),(x, p, \theta)^{T}$ the coordinates of the detector, and $\mathbf{r}=(x, y, z)^{T}$ the coordinates in real space as explained in Fig. 2. In particular the $x$-axis is set parallel to the tilt axis in order to reduce the $3 \mathrm{D}$ reconstruction to a slice by slice 2D tomographic reconstruction problem in planes perpendicular to the tilt axis.

Following the mathematical foundations of tomography (e.g. [6]), the collection of projected potentials (11) under different angles $\theta$ represents the Radon transform $\hat{V}=\mathcal{R}\{V\}$ of $V$. The inversion of this transformation (inverse Radon transformation) then yields the potential $V=\mathcal{R}^{-1}\{\hat{V}\}$ forming the basis of EH tomography (EHT). The proof-of-concept for EHT dates back to the work of Lai et al. [7], however, quantitative reconstructions [810] were delayed for a long time mainly for two reasons: first, the instrumental demands, such as specific tomography specimen holders allowing ultrahigh tilt angles, nanometer precise computer-controlled goniometers as well as powerful computers had to be developed. Second, the EHT procedure is very comprehensive and time consuming.

The most notable experimental limitation of virtual all electron-microscopic tomographic techniques is the limited tilt range of usually $\pm 70^{\circ}$ instead of $\pm 90^{\circ}$. This leads to a loss of information, visible as "missing wedge" in the Fourier transform of the tomogram (see e.g. [11]). In real space, this corresponds to a reduced resolution in the tomogram in the direction of the missing wedge. Moreover, dynamical scattering effects, tilt axis misalignment, phase unwrapping failures and shot noise introduce errors in the projected data that can amplify upon reconstruction because of the (mildly) ill-conditioned nature of the Radon transform [6]. Similar to other tomographic techniques, large and ongoing efforts are therefore put into developing regularization schemes

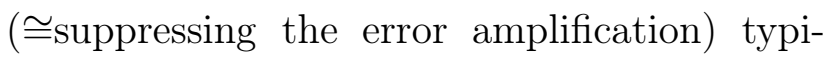
cally involving auxiliary conditions such as (eu-

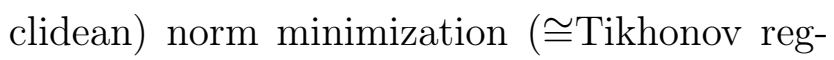
ularization [12]), total variation minimization (@generalized Tikhonov regularization [13]) or signal range restriction (discrete tomography [14]). Since such conditions typically introduce an additional regularization error, the art of regularization consists of minimizing the total error of both original and regularization error. That task is further complicated by the fact 
that these errors are generally unknown [15]. It is therefore always advantageous to use preferably error-free conditions based on real physical properties of the potential such as physical spatial constraints (e.g. [8]). That can be due to outer or internal boundaries of nanoparticles or symmetries imposed by the crystal structure.

How spatial constraints can be used to improve the tomographic reconstruction will be discussed in the following section. We will furthermore emphasize the importance of sampling, that is, the relation between detector resolution, number of projection angles and reconstructed object features, for the design of optimal reconstruction algorithms. The obtained improvement will be valuable for all types of tomography in spite of our particular application to EHT. Our second focus are improving methods for phase unwrapping, dynamic scattering correction and tilt series alignment, mainly dedicated to EHT. The combination of which will significantly improve EHT as 3D potential reconstruction method.

Using the optimized reconstruction procedure we successfully retrieved the $3 \mathrm{D}$ potential of a $\langle 111\rangle$-oriented GaAs- $\mathrm{Al}_{0.33} \mathrm{Ga}_{0.67}$ As coreshell nanowire (NW) grown by metal-organic vapor phase epitaxy (MOVPE) through a $\mathrm{Au}$ nanoparticles (NP) as metal catalyst (see Ref. [16, 17]). The structure provides a wide range of electrostatic potentials with sharp interfacesas well as long rang gradients. The AlGaAs shell contains also self-assembled Al segregations and local alloy fluctuations due to the different $\mathrm{Ga}$ and $\mathrm{Al}$ adatom mobilities on the nanowire surface [18, 19]. Note that these features are crucial for envisaged applications of semiconductor NW in future nano-scaled electronic, optoelectronic, and photovoltaic devices [20 22]. Moreover, we previously investigated this system with less developed EHT techniques [17], rendering it the ideal test case for verifying the achieved progress.

\section{ALGEBRAIC HOLOGRAPHIC TOMOGRAPHY}

The general workflow of EHT consisting of hologram tilt series acquisition, phase reconstruction from hologram tilt series; phase tilt series alignment and tomographic reconstruction is depicted in Fig. 2 using the example of the GaAs-AlGaAs NW. All steps have been widely automated, not only to decrease the amount of time from initially $\mathcal{O}(10 \mathrm{~h})$ to presently $\mathcal{O}(1 \mathrm{~h})$ (see [10] for details), but also to increase the reproducibility and quality of the obtained data. Thus, the automated workflow is an important prerequisite towards widespread application. The holographic tilt series of an individual NW with an entire diameter of $300 \mathrm{~nm}$ (80 nm core, $110 \mathrm{~nm}$ shell) was recorded in a range from $-69^{\circ}$ to $+72^{\circ}$ in $3^{\circ}$ steps at the FEI Titan 80-300 Berlin Holography Special TEM using the THOMAS software package [10]. The TEM was operated at $300 \mathrm{kV}$ acceleration voltage in aberration corrected Lorentz mode that provides a resolution of about $2 \mathrm{~nm}$. The holograms were acquired 
with a double biprism setup with a field of view of $1 \mu \mathrm{m}$ and a fringe spacing of $3 \mathrm{~nm}$. The object exit wave tilt series has been reconstructed from the object holograms and corresponding empty holograms applying a Butterworth filter of $0.13 \mathrm{~nm}^{-1}$ FWHM to separate the side band (see e.g. in [23] for further details of the holographic method). This was performed automatically for the entire tilt series. Fig. 1 shows a typical example of an holographically reconstructed projected potential of the NW. To highlight the effects in the vacuum region due to beam charging and remaining phase wedges due to the sideband alignment we also show the outer region in Fig. 1. Here, one observes a small charging field accumulating around $50 \mathrm{Vnm}$ at the edge of the NW and dropping quickly to $-50 \mathrm{Vnm}$ towards the rim of the reconstruction volume. Considering that the whole NW has a diameter of $300 \mathrm{~nm}$ this corresponds to a surface potential due to charging of around $0.3 \mathrm{~V}$. To test its influence on the tomographic reconstruction we performed the latter with and without constraining the complete vacuum to $0 \mathrm{~V}$ as stated in the results section below.
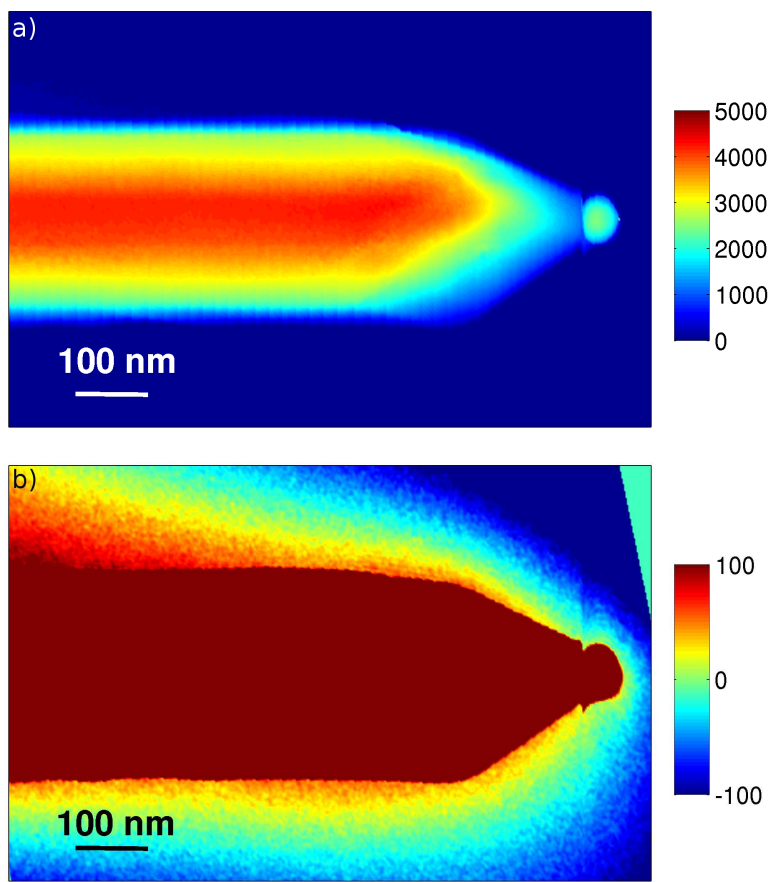

Figure 1: Examplary projected potential (in Vnm) reconstructed from a hologram of the tilt series (at $30^{\circ}$ tilt angle) depicted at two different colour scales to illustrate the magnitude of the charging. On top the complete potential reachig several $\mathrm{kVnm}$ in the NW is depicted. The charging field below is approximately 2 order of magnitude lower and is also slightly superimposed by an additional phase wedge from the imperfect alignment of the sideband. The zero potential region on the top right stems from the alignment of the tilt axis along $x$.

Following holographic wave reconstruction and reorientation of the tilt axis along the $x$ axis, three important processing steps determine the accuracy of the subsequent tomographic reconstruction: (1) The phase needs to be unwrapped to obtain the projected potential from the reconstructed waves. The unwrapping is problematic if the sampled phase 
difference between two pixels exceeds $\pi$ [24], a situation often present in noisy data with sharp thickness jumps at specimen edges. We tackled this problem by extending the original 2D unwrapping performed at each holographic reconstruction to $3 \mathrm{D}$ with the tilt angle as third dimension (i.e. the whole tilt series). That is possible because of the continuous dependency of the phase on the tilt angle and yielded accurate projected potentials even at the Au catalyst. The next step (2) consists of suppressing dynamical scattering artifacts producing oscillations of the phase close to zone axis conditions (Fig. 2a)). The latter could be largely removed by normalizing the average of the projected potentials at all angles to the same value, in agreement with a dynamical correction factor approach [25]. (3) Finally, this data is aligned around a common tilt axis with the center of mass method. This alignment was performed slice by slice for sampling reasons discussed below. The tilt series ready for tomographic reconstruction is depicted in Fig. 2b. Besides the substantial improvement of the data one observes a 6-fold symmetry in the core-shell region reducing to a 3-fold symmetry in the tapered section below the Au NP (corroborated by azimuthal crosscorrelation in Fig. 2b).

Now the actual tomographic reconstruction starts. The variety of reconstruction techniques can be separated into filtered, Fourier and algebraic algorithms, which coincide in the limit of infinitely fine sampling. Fourier methods and filtered techniques, such as Weighted
Back Projection (WBP) [26, 27] or Weighted Simultaneous Reconstruction Techniques (WSIRT) [28] are based on numerically efficient and fast adaptions of analytic Radon transformation formulas. Algebraic reconstructions consider the sampled Radon transformation as a large system of linear equations which are typically inverted by iterative algebraic techniques. Here, we will implement and further develop the latter because of its higher accuracy and flexibility at the cost of only minor reduction in speed.

In Fig. 3 the main idea behind algebraic reconstruction is illustrated: Here, the set of all sampled projections with index $m$ is the algebraic version of the Radon transform $\hat{V}_{m}=$ $\mathcal{R}_{m n} V_{n}$ of the deliberately sampled potential (index $n$ ). The Radon matrix is usually sparse and tomographic reconstruction corresponds to finding a (generalized) inverse to the latter. Although sampling the position space by a the Cartesian grid is by far dominant in applications we will show in the following that the polar grid is the better choice indeed:

(i) First of all there is a deep connection between the polar grid and sampling theorems valid for $2 \mathrm{D}$ Radon transformations. The latter state that tomographic reconstruction from finite tilt angles requires azimuthally bandlimited data [6] (see also Supplementary Information). From that it follows that the axis with the smallest azimuthal band limit not only determines the number of required tilt angles in the experiment but also the number of azimuthal grid points in the reconstruction 
a) Holography

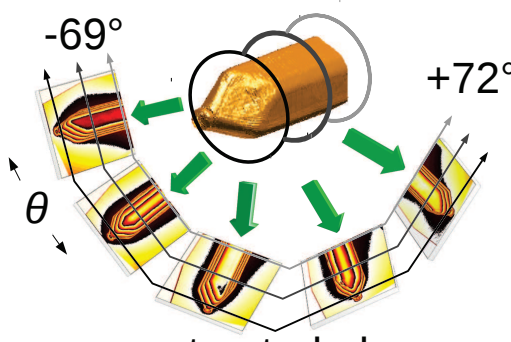

reconstructed phases

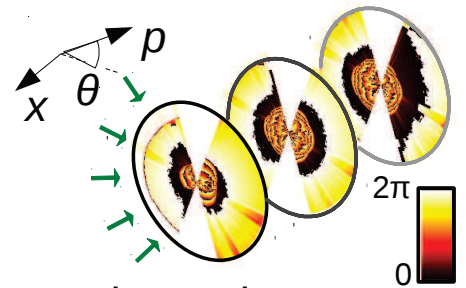

phase sinogram b) Preprocessing
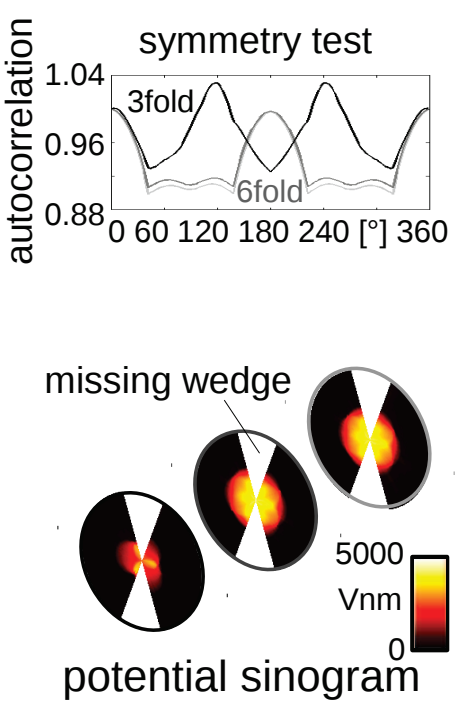

c) Tomography
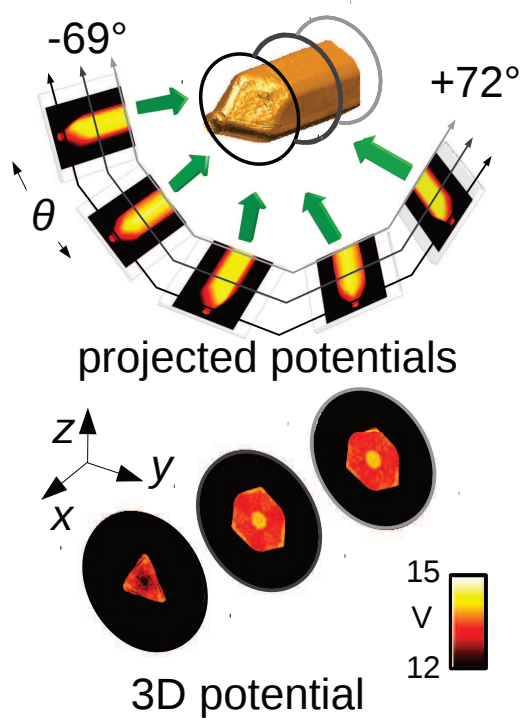

Figure 2: Holographic tomographic principle: a) Holographic tilt series (top) and selected sinograms (bottom). b) Rotational symmetry test (top) on preprocessed projected potential data (bottom). The 6-fold (hexagonal) symmetry of the NW is partly suppressed by the 2-fold symmetry of the missing wedge. c) Tomographic reconstruction (top) and reconstructed potential (bottom). Throughout the figure the 5 green arrows denote a set of angles $\left(-66^{\circ},-33^{\circ}, 0^{\circ}, 33^{\circ}, 66^{\circ}\right)$ and 3 deliberately chosen slices at $x=100,400,700 \mathrm{~nm}$ are highlighted by black, dark gray and light gray lines and frames.

on a polar grid. For example, a radially symmetric object (e.g. a homogeneous cylinder) aligned along the symmetry axis can be reconstructed from a single projection only [29]. Accordingly, some large annular pixels with radial dimensions determined by the radial resolution are sufficient when reconstructing this function on a polar grid centered around the symmetry axis. Obviously the number of Cartesian pixels would have been much larger in this case. By aligning the tilt series slice-wise around the center of mass we closely approximate the 3-fold symmetry axis of the NW facilitating a beneficial use of the polar grid in terms of minimizing pixel numbers in posi- tion space. By this, memory requirements and computing time for matrix inversion (typically scaling with (matrix dimension) ${ }^{2 \ldots 3}$ ) could be greatly reduced. (ii) It is immediately obvious that the above noted spatial constraints can be straight forwardly implemented by shaping the projected and reconstructed pixels correspondingly. Fig. 3b) illustrates sampling schemes for $n$-fold rotationally symmetric objects, azimuthal band limited objects and segmented objects. Indeed, one can deliberately combine different constraints. Beside these two main advantages we note that the radial grid exploits the whole circular reconstruction area. For the (generalized) matrix inversion it- 


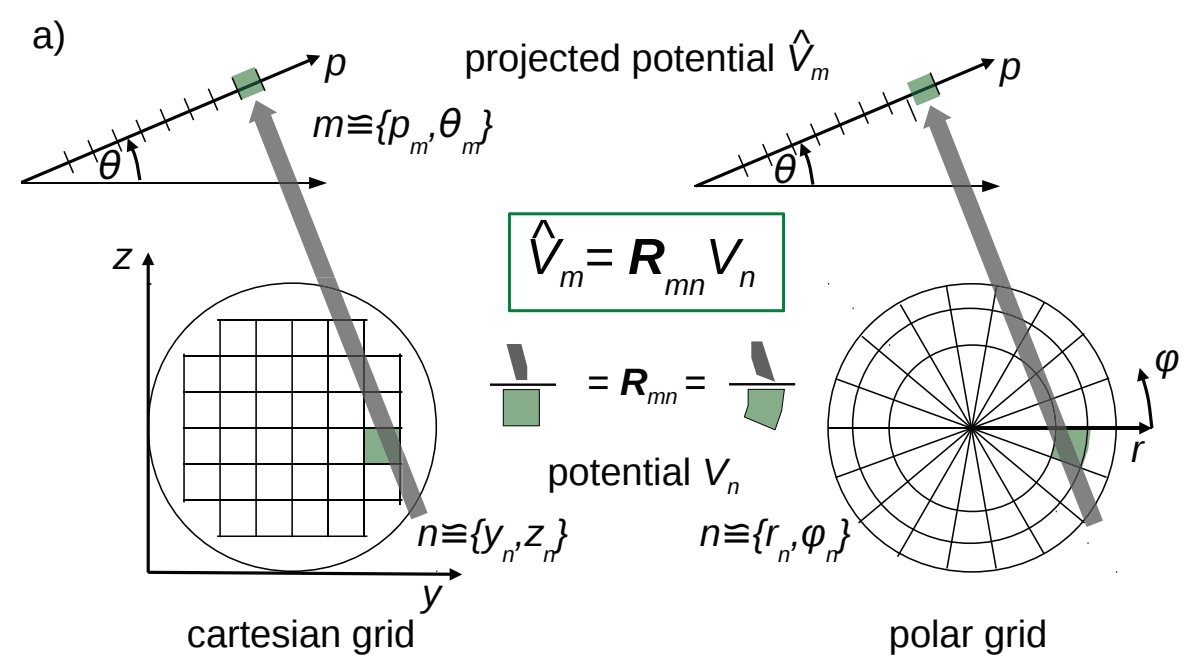

b) spatial constraints
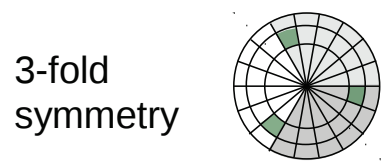

azimuthal

bandlimited

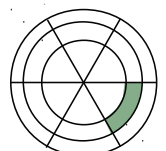

segmented

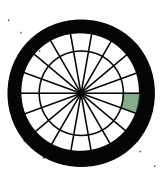

Figure 3: Algebraic tomography and sampling: a) A deliberately chosen sampling (left: Cartesian, right: polar) with sampling index $n$ is projected under a certain angle $\theta_{m}$ on the detector pixel $p_{m}$ with the projection weight of the reconstructed pixel $n$ determined by the normalized covering of the projection ray. b) Three examples of spatial constraints applied to the polar sampling scheme from top to bottom: 3 -fold symmetric projection of 3 symmetric pixels into corresponding detector pixels; azimuthal band-limited projection facilitating reduction of azimuthal sampling points; deliberate exclusion of potential free region (e.g. vacuum) from Radon matrix.

self a large number of numerical methods has been developed. For tomography the Kacz-

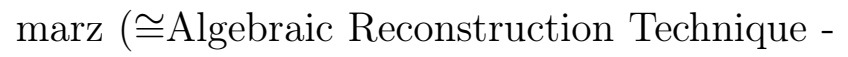
ART) and related methods (e.g. Sequential Iterative Reconstruction Technique - SIRT) are very popular [6], probably due to its particularly easy implementation and built-in quasi Tikhonov regularization parametrized by the number of iterations [15]. The latter refers to a a growing reconstruction of larger spatial frequency components at higher iteration numbers, implying a growth of spatial resolution at cost of increasing noise. However, the Kaczmarz method is not seeking for the optimal (fastest) iteration and is not optimized for sparse matrices such as the Radon matrix.
Therefore, we use a conjugate gradient method implemented in the LSQR algorithm [30] to reduce significantly the number of iterations (due to a weaker quasi Tikhonov regularization) and thus computing time.

\section{RESULTS}

We present results from a tomographic reconstruction incorporating a 3-fold symmetry constraint and 10 LSQR iterations on a $13800 \times 11700$ Radon matrix for a polar grid This choice is motivated by the good reconstruction quality (good spatial resolution at acceptable noise) containing all important potential features. We emphasize, however, that the regularization strength $(\cong$ the number of it- 
erations) can be deliberately varied; therefore we supplement reconstructions with 5 and 15 iterations and a reconstruction without symmetry constraint (see Supplementary Information). We furthermore note that a small charging of the NW could be observed through stray fields in the vacuum (see Fig. 1). Its influence on the reconstruction was tested to be around $0.1 \mathrm{~V}$ by reconstructing with and without constraining the vacuum to $0 \mathrm{~V}$ in the reconstruction. The reconstructed $3 \mathrm{D}$ potential of the GaAs- $\mathrm{Al}_{0.33} \mathrm{Ga}_{0.67} \mathrm{As}$ NW, which is illustrated by means of isosurfaces and $y z$-slices in Fig. 4b shows a slightly deformed 6-fold symmetry in the core-shell region corroborating the symmetry test performed on the projection data (Fig. 2b). We emphasize that due to the symmetry constraint missing wedge artifacts are now completely absent (see [17] for comparison); it would even be possible to use a trunctated tilt series interval of $60^{\circ}$ here. In total, the achieved signal precision of about $0.2 \mathrm{~V}$ (determined from the standard deviations of the corresponding homogeneous potential region along $x$ ) and spatial resolution of $6 \mathrm{~nm}$ (determined from the FWHM of the potential gradient at the NW boundary) is mainly limited by the holographic imaging process and not the EHT method; and therefore allows detecting the following features in $3 D$.

The Au NP at the NW tip has an av-

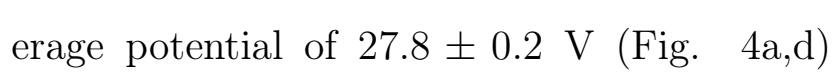
which agrees very well with the theoretical value of $28 \mathrm{~V}$ obtained by multiplying the DFT value $30.1 \mathrm{~V}$ [31] with a dynamical scatter- ing correction factor of 0.93 [25]. The same holds for the average potential of the GaAs core $\left(V_{\exp }=13.9 \pm 0.1 \mathrm{~V}, V_{\text {theo }}=14.19 \mathrm{~V}\right.$ [32]) and the $\mathrm{Al}_{0.33} \mathrm{Ga}_{0.67} \mathrm{As}$ shell $\left(V_{\exp }=\right.$ $13.4 \pm 0.2 \mathrm{~V}, V_{\text {theo }}=0.33 \cdot 12.34 \mathrm{~V}+0.67$ $14.19 \mathrm{~V}=13.58 \mathrm{~V}$ [32]). [36] We furthermore observe an axial decay of the potential from the $\mathrm{Au}-\mathrm{Al}_{0.33} \mathrm{Ga}_{0.67} \mathrm{As}$ interface over approximately $100 \mathrm{~nm}$ comprising $0.5 \mathrm{~V}$ (Fig. 4d), which could indicate a Fermi-Level-pinned Metal-AlGaAs junction or a varying chemical composition. In particular the appearance of a small potential $(V=12.5 \pm 0.1 \mathrm{~V})$ region directly above the GaAs core (Fig. 4d) indicates the formation of a AlAs alloy reported for similar GaAs- $\mathrm{Ga}_{c} \mathrm{In}_{1-c} \mathrm{P}$ core-shell NWs [33]. The most remarkable features are characteristic 3-fold symmetric lines of reduced potential along \{112\}-directions of the NW (Fig. 4e). This observation is in striking agreement with the facet-dependent Al-segregation recently reported for this system [18, 34], and ascribed to polarity-driven surface reconstruction during AlGaAs shell growth. Noteworthy, in Refs. [18, 34] Al-enrichment along \{112\}-facets was detected in the shell by means of $2 \mathrm{D}$ crosssectional STEM analysis and the determined $\mathrm{Al}$ concentration enhancement to $c_{A l} \approx 0.67$ agree with our value $c_{A l}=0.55 \pm 0.5$ when taking into account a further increase at higher iteration numbers $(\cong$ higher spatial resolution, see Supplementary Information).

A close inspection of the $3 \mathrm{D}$ potential additionally revealed fluctuations of the Alenrichment around the above mean value. 

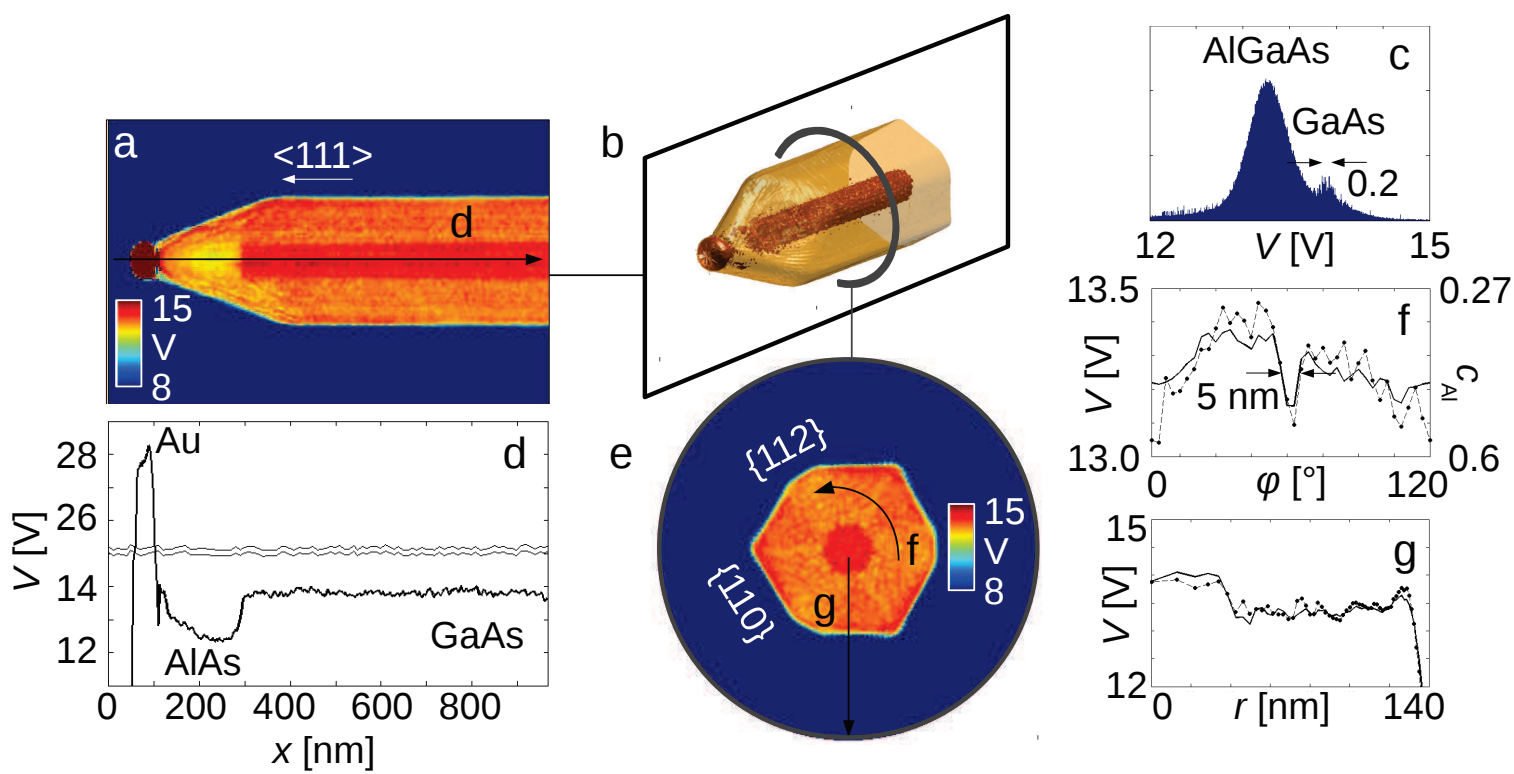

Figure 4: Reconstructed 3D potential of GaAs- $\mathrm{Al}_{0.33} \mathrm{Ga}_{0.67} \mathrm{As}$ core shell NW: The isosurfaces (b) at 9 and $13.75 \mathrm{~V}$ illustrate the core-shell morphology corroborated by the double peaks in the corresponding histogram (c). The $z x$-plane (a) with corresponding linescan (d) on the left shows the sequence Au, AlAs and GaAs unraveled through their different MIPs. The projected yz-plane (e) on the right with corresponding linescans in azimuthal (f) and radial (g) direction shows the core-shell structure with local $\mathrm{Al}$ accumulation identified through characteristic potential reduction in the azimuthal scan. Here, the corresponding $\mathrm{Al}$ concentration $c_{A l}$ determined from Vegard's Law is shown on the right $\mathrm{y}$-scale. The thick lines in (f) and (g) indicate averaged values from the whole hexagonal region of the NW.

We ascribe them to modulations of the local length scale. Secondly, we observe a complete faceting of the core as demonstrated in Fig. rotation of about $30^{\circ}$ between $x=345.5 \mathrm{~nm}$ 5. where we show cross-sections from the un- to $x=347.6$ possibly introduced by a twin perturbed middle part (i.e. far away from boundary or a switch from $\{110\}$-facets to the tapered region) of the NW. The depicted $\{112\}$-facets. These changes could be observed set of cross-sections clearly reveals a chang- along the whole NW and are subjected to influing facet structure. Two effects can be distin- ence the growth of the shell structure in general guished. First there is a change in facet length, and the Al-accumulation in particular.

e.g. observable in the sequence $x=335 \mathrm{~nm}$ to $x=345.5 \mathrm{~nm}$. That effect has been reported in Ref. [35] and was there explained by alternating $\{111\}$-nanofacets accumulating to the six-fold $\{110\}$-facets observed on a larger 

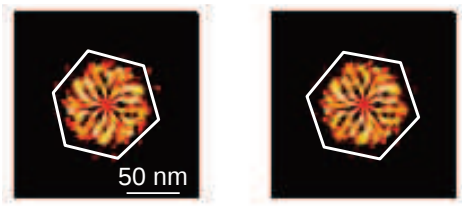

$\mathrm{x}=337.1 \mathrm{~nm}$

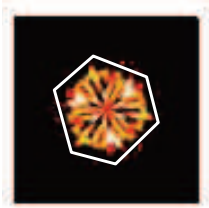

$\mathrm{x}=341.3 \mathrm{~nm}$

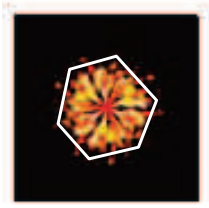

$\mathrm{x}=347.6 \mathrm{~nm}$

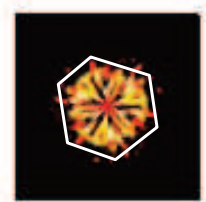

$\mathrm{x}=343.4 \mathrm{~nm}$

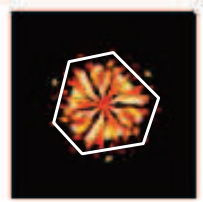

$\mathrm{x}=349.7 \mathrm{~nm}$

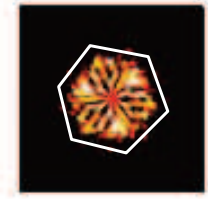

$\mathrm{x}=339.2 \mathrm{~nm}$

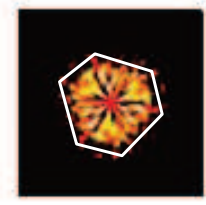

$\mathrm{x}=345.5 \mathrm{~nm}$

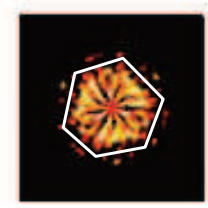

$\mathrm{x}=351.8 \mathrm{~nm}$

Figure 5: GaAs-core showing a modulated facet structure. White frames are drawn around the core to indicate the core facets. The color range was restricted to an interval between 13.5 and 14.5 $\mathrm{V}$ to highlight the core region.

We again emphasize that the above features have been reconstructed in 3D for the first time (see Ref. [17] for comparison), i.e. quantitative compositional changes along $x, y$ and $z$ are revealed in parallel without the need to destructively prepare special cross-sections with possibly modified surfaces from the NW.

\section{SUMMARY}

In summary we demonstrated quantitative 3D electrostatic potential reconstruction with unprecedented accuracy and spatial resolution by a number of separate improvements implemented into electron holographic tomography

(EHT). Most importantly, we developed automated acquisition and wave reconstruction schemes, procedures for 3D phase unwrapping, dynamic scattering correction, as well as optimal polar and symmetry adapted sampling. The latter two are beneficial to all tomographic techniques as a route towards faster and more accurate reconstructions. For the particular case of EHT our techniques facilitate reconstruction of nanoscale potentials such as originating from band bending or elemental segregation where spatial and signal resolution are critical. Similarly, 3D magnetic field reconstruction can be simplified by segmentation of the reconstruction volume into particle support and vacuum with the latter facilitating a reconstruction without the critical subtraction of the electrostatic phase shift.

\section{ACKNOWLEDGMENTS}

AL, DW, and HL acknowledge financial support from the European Union under the Seventh Framework Programme under a contract for an Integrated Infrastructure Initiative. Reference 312483 - ESTEEM2. 8. SS is funded by the European Union (ERDF) and the Free State of Saxony via the ESF project 100087859 ENano. TN acknowledges financial support from the DFG within SFB 787 and INST 131/508-1. 
D. Smith, in Encyclopedia of Nanoscience and Nanotechnology, edited by H. Nalwa (American Scientific Publishers, 2004), vol. 3, pp. 41-100.

[3] H. Lichte and M. Lehmann, Reports on Progress in Physics 71, 016102 (2008).

[4] P. A. Midgley and R. E. Dunin-Borkowski, Nature materials 8, 271 (2009).

[5] G. Matteucci, G. Missiroli, and G. Pozzi, in Electron Microscopy and Holography II, edited by P. W. Hawkes (Elsevier, 2002), vol. Volume 122, pp. 173-249.

[6] F. Natterer, The mathematics of computerized tomography, vol. 32 of Classics in applied mathematics (Society for Industrial and Applied Mathematics, Philadelphia, 2001), ISBN 0898714931.

[7] G. Lai, T. Hirayama, K. Ishizuka, and A. Tonomura, Journal of Applied Optics 33, 829 (1994).

[8] A. C. Twitchett-Harrison, T. J. V. Yates, S. B. Newcomb, R. E. Dunin-Borkowski, and P. A. Midgley, Nano Letters 7, 2020 (2007).

[9] T. Fujita and M. Chen, Journal of Electron Microscopy 58, 301 (2009).

[10] D. Wolf, A. Lubk, H. Lichte, and H. Friedrich, Ultramicroscopy 110, 390 (2010).

[11] P. A. Midgley and M. Weyland, Ultramicroscopy 96, 413 (2003).

[12] A. N. Tikhonov and V. Y. Arsenin, Solution of Ill-posed Problems. (Washington: Winston \& Sons, 1977).

[13] B. Goris, W. Van den Broek, K. Batenburg, H. Heidari Mezerji, and S. Bals, Ultrami- croscopy 113, 120 (2012).

[14] K. Batenburg, S. Bals, J. Sijbers, C. Kübel, P. Midgley, J. Hernandez, U. Kaiser, E. Encina, E. Coronado, and G. V. Tendeloo, Ultramicroscopy 109, 730 (2009).

[15] P. C. Hansen, Rank-Deficient and Discrete Ill-Posed Problems: Numerical Aspects of Linear Inversion (SIAM, Philadelphia,, 1998).

[16] P. Prete, F. Marzo, P. Paiano, N. Lovergine, G. Salviati, L. Lazzarini, and T. Sekiguchi, Journal of Crystal Growth 310, 5114 (2008).

[17] D. Wolf, H. Lichte, G. Pozzi, P. Prete, and N. Lovergine, Applied Physics Letters 98, 264103 (pages 3) (2011).

[18] D. Rudolph, S. Funk, M. Doblinger, S. Morkötter, S. Hertenberger, L. Schweickert, J. Becker, S. Matich, M. Bichler, D. Spirkoska, et al., Nano Letters 13, 1522 (2013).

[19] M. Heiss, Y. Fontana, A. Gustafsson, G. Wüst, C. Magen, D. D. O'Regan, J. W. Luo, B. Ketterer, S. Conesa-Boj, A. V. Kuhlmann, et al., Nature materials 12, 439 (2013).

[20] T. Bryllert, L.-E. Wernersson, T. Löwgren, and L. Samuelson, Nanotechnology 17, S227 (2006).

[21] E. M. Gallo, G. Chen, M. Currie, T. McGuckin, P. Prete, N. Lovergine, B. Nabet, and J. E. Spanier, Applied Physics Letters 98, 241113 (2011).

[22] P. Krogstrup, H. I. Jorgensen, M. Heiss, O. Demichel, J. V. Holm, M. Aagesen, J. Ny- 
gard, and A. Fontcuberta i Morral, Nat Photon $\mathbf{7}, 306$ (2013).

[23] M. Lehmann and H. Lichte, Microscopy and Microanalysis 8, 447 (2002).

[24] D. C. Ghiglia and M. D. Pritt, TwoDimensional Phase Unwrapping (Wiley, 1998).

[25] A. Lubk, D. Wolf, and H. Lichte, Ultramicroscopy 110, 438 (2010).

[26] P. Gilbert, Proc. R. Soc. Lond. B. 182, 89 (1972).

[27] M. Radermacher, in Electron Tomography, Methods for Three-Dimensional Visualization of Structures in the Cell, edited by J. Frank (Springer, Berlin, 2006), pp. 245-274.

[28] D. Wolf, A. Lubk, and H. Lichte, Ultramicroscopy 136, 15 (2014).

[29] V. Grillo and F. Rossi, Ultramicroscopy 125, 112 (2013).

[30] C. C. Paige and M. A. Saunders, ACM Transactions on Mathematical Software 8, 195 (1982).

[31] M. Schowalter, A. Rosenauer, D. Lamoen,
P. Kruse, and D. Gerthsen, Applied Physics Letters 88, 232108 (2006).

[32] P. Kruse, M. Schowalter, D. Lamoen, A. Rosenauer, and D. Gerthsen, Ultramicroscopy 106, 105 (2006).

[33] N. Sköld, L. S. Karlsson, M. W. Larsson, M.-E. Pistol, W. Seifert, J. Trägardh, and L. Samuelson, Nano Letters 5, 1943 (2005).

[34] C. Zheng, J. Wong-Leung, Q. Gao, H. H. Tan, C. Jagadish, and J. Etheridge, Nano Lett. 13, 3742 (2013).

[35] J. Johansson, L. S. Karlsson, C. P. T. Svensson, T. Mårtensson, B. A. Wacaser, K. Deppert, L. Samuelson, and W. Seifert, Journal of Crystal Growth 298, 635 (2007).

[36] The small discrepancy could be due to small imperfections of the generalized-gradient approximation of exchange-correlation in the theoretic calculations, the choice of the phase reference in the holographic experiment or the small charging field. 